\title{
Principles and Available Hardware in DECT
}

\author{
Ezhilmathi Alavandar ${ }^{1}$ Venkatesh Kasi Arunachalam ${ }^{1}$ Niyas Narappulan ${ }^{1}$ Gowtham S. Mahadevan ${ }^{1}$ \\ Ravindar Kashyap ${ }^{1}$ Pankaj Mehta ${ }^{1}$ Mathew Cherian ${ }^{1}$ \\ 1 Department of Radiology, Kovai Medical Center and Hospital, \\ Coimbatore, India \\ J Gastrointestinal Abdominal Radiol ISGAR 2022;5:76-84.

\begin{abstract}
Address for correspondence Venkatesh Kasi Arunachalam, DMRD, DNB, FRCR, Department of Radiology, Kovai Medical Center and Hospital, 99, Avinashi Road, Peelamedu, Indira Nagar, Civil Aerodrome Post, Coimbatore, Tamil Nadu 641014, India

(e-mail: drkasivenkatesh@yahoo.co.in).
\end{abstract}
Abstract
Keywords
- CT
- diagnostic imaging
- DECT
- attenuation
- applications
- principle

Computed tomography (CT) has undergone a phenomenal evolution since its introduction in 1971 and has revolutionized diagnostic radiology. It is now the cornerstone of diagnostic imaging and has become an inevitable part of the management of patients. Among all the advancements and breakthroughs witnessed over the years, the most recent and most advanced is the dual-energy CT (DECT), also known as spectral CT, introduced in 2006. In DECT, two datasets are obtained by scanning with two different energy spectra (low and high energy). The difference in attenuation can differentiate materials with different elemental compositions but similar attenuation in single-energy CT. Therefore, it has widespread clinical applications based on its potential for material decomposition and virtual monoenergetic imaging. In this review, the principle and hardware of DECT will be presented with an overview of its clinical applications.

\section{Introduction}

Computed tomography (CT) was pioneered in the early 1970s by Sir Godfrey N. Hounsfield, an English electrical engineer. The first CT scanner was a two-slice machine designed to scan the brain with a pencil beam in a step and shoot mode. Following that, whole body fan-beam CT scanners for general radiology were introduced. Helical CT acquisition came into the arena in the early 1990s with the introduction of slip ring technology. Volume date acquisition was possible due to continuous table translation in helical CT.

In 1998 multislice CT systems were introduced by all major vendors with simultaneous acquisition of four slices per rotation. ${ }^{1}$ This breakthrough resulted in vast potential for increased isotropic voxel resolution, leading to precise reformations and faster scanning times, resulting in accurate multiphase imaging and angiography studies. Hence, there was a race for more slices in the consequent years, which resulted in the advent of the eight-slice CT system in 2000, 16-slice, 32- slice, 64-slice henceforth. Furthermore, the sub-millimeter spatial resolution of recent CT scanners and faster gantry rotation times up to 0.375 second have made electrocardiogram-gated cardiac studies and angiography feasible with increased image quality. ${ }^{1}$

However, the substances with different chemical compositions can show similar attenuation (Hounsfield unit) in single-energy CT. Until recently, there was no tool for material differentiation and tissue characterization. This problem was solved by introducing dual-energy CT (DECT) in 2006 by Siemens (Somatom definition). DECT has its roots as early as 1973 when Godfrey Hounsfield described that two pictures were taken of the same slice at $100 \mathrm{kV}$ and the other at $140 \mathrm{kV}^{2}$ This was also further investigated by Alvarez and Macovski in 1976. They showed that energy-dependent information could be obtained from the polychromatic X-ray spectrum by separating the measured attenuation coefficients into their contributions from photoelectric and published online February 17, 2022
DOI https://doi.org/ $10.1055 / \mathrm{s}-0042-1742772$. ISSN 2581-9933.

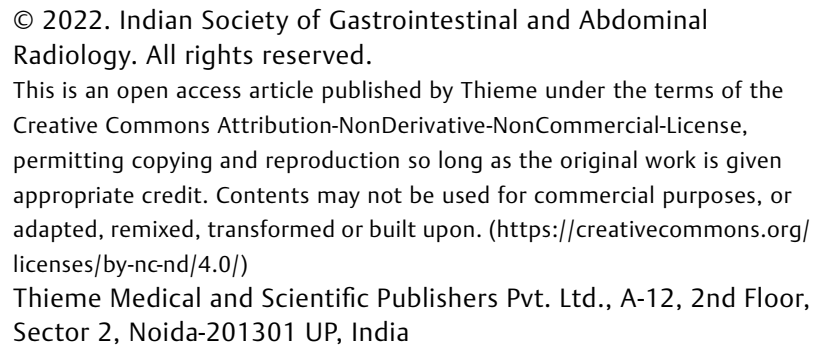


Compton effects. ${ }^{2-4}$ Due to the technological limitations at that time, the successful application of these investigations was not feasible. The principle, techniques of acquisition with their advantages and limitations, and an overview of image processing algorithms and clinical applications will be presented in this review.

\section{Principle}

DECT, also known as spectral CT, uses two different energy spectra (low and high energy) for imaging to make material decomposition and tissue characterization possible. Due to two different energy spectra, data of tissue attenuation at these different energies can be analyzed, leading to material differentiation. DECT is a subset of spectral CT that uses two $\mathrm{X}$-ray spectra for imaging which means that there is potential for acquiring more than two energy levels by using energy resolving photon-counting detectors, the subject of ongoing research, which can result in multi-energy CT. ${ }^{2,5}$

\section{Energy and Material Dependence}

Materials with different elemental compositions can have similar attenuation in CT depending on their mass density. For their differentiation, it is necessary to use a second X-ray spectrum for imaging. This provides an additional attenuation measurement, and the difference in attenuation between the two energy spectra is because of the energy and material dependence. Usually, in DECT, the two energy spectra used are commonly in the range of 80 to $90 \mathrm{kV}$ (low energy) and 140 to $150 \mathrm{kV}$ (high energy). Due to the maximum difference and better spectral separation, the standard peak energies are 80 and $140 \mathrm{kV} .^{5,6}$

For heavier patients requiring greater penetrance, 90 or $100 \mathrm{kV}$ can be used for the lower energy range. Energy lower than $80 \mathrm{kV}$ is not advisable except for use in pediatric patients because most of the photons in this energy range are absorbed by the body. Due to suboptimal soft-tissue resolution and increased radiation dose, higher energies greater than $150 \mathrm{kV}$ could not be used. Filters can be used with the low- or high-energy spectra to remove the overlap resulting in superior spectral separation.

$\mathrm{X}$-ray attenuation by the material is accounted by the processes involved in the interaction of photons with matter, out of which photoelectric and Compton effects play a significant role. The Compton effect is minimally energydependent and depends mainly on the electron density of the material. The photoelectric effect is strongly energydependent (probability more at a low-energy range) and is critical for material differentiation. The photoelectric effect also increases with the atomic number of the material. Most of the tissues in the body are made predominantly of lower atomic number atoms like hydrogen, carbon, and nitrogen and, hence, show a weak photoelectric effect except for calcium and magnesium, which may show a more substantial effect. On the contrary, the contrast materials used in imaging, i.e., iodine and barium, due to their higher atomic number and K-edge effect, have a strong photoelectric effect resulting in strong spectral contrast in DECT between the rest of the atoms of the body and the contrast material. ${ }^{6}$ The difference in spectral properties of the materials/tissues, i.e., the difference in atomic numbers, and the energy dependence, plays a pivotal role in optimal spectral contrast in DECT images and thus in its clinical applications.

\section{Technology in the Acquisition of DECT}

The acquisition of two energy spectra can be done in varying ways and is broadly classified as source-based and detectorbased acquisitions (-Fig. $\mathbf{1}$ ).

\section{Source-Based Acquisition}

\section{Dual-Source DECT (Ds DECT)}

Two X-ray tubes with corresponding detectors are mounted orthogonally at $90^{\circ}$ with different tube potentials, usually 80 and $140 \mathrm{kV}$. Data are obtained by the simultaneous acquisition of these two X-ray tubes operating at a different potential. The tube voltage and current can be adjusted independently for both tubes resulting in optimal spectral contrast. Prefiltration can be done by adding a filter for both tubes separately, enabling better spectral separation and noise reduction. ${ }^{5,7}$ More importantly, Ds DECT enables simultaneous imaging of a slice at two different kilovolts without any temporal offset. ${ }^{6}$ However, projection data are $90^{\circ}$ out of phase because of the angular offset of the X-ray tubes within the gantry, and so dual-energy processing algorithms are performed in the image domain. ${ }^{5,7}$ This is the technique developed by Siemens Healthineers, Germany.

\section{Limitations}

- Small field of view (FOV): To accommodate two X-ray tubes with detectors within the gantry, the second detector is restricted to a smaller FOV. The scan FOV for the Siemens Ds DECT is as follows: first generation-26 cm, second generation $-33 \mathrm{~cm}$, and third generation $-35.6 \mathrm{~cm} .^{7}$

- Cross-scattered radiation: Scattered radiation from one X-ray tube reaches the orthogonal detector of the other X-ray tube, leading to spectral distortion. However, it can be rectified by the appropriate algorithm. ${ }^{2,6-8}$

\section{Rapid Kilovolt Switching}

X-ray tube rapidly switches between the low and high voltages in a short time ( $<0.2$ milliseconds) within a single gantry rotation, enabling high temporal resolution and minimal offset between the projections of two energy spectra. So, material decomposition can be performed in the projection domain, thereby reducing the beam-hardening artifacts. ${ }^{2,8,9}$ This technology was introduced by General Electric Healthcare, United States. Tube current cannot be modulated for each tube potential in this configuration of rapid voltage switching. Alternatively, asymmetric sampling can be implemented for optimizing tube current by allowing a prolonged exposure and higher current for low kilovolts (in the ratio of $80 / 140=60 \%$ / $40 \%$ ) or by acquiring two low-voltage projections for every single high-voltage projection. ${ }^{2,5,8}$ Spectral filtration is also not technically feasible because the same X-ray source is used for both voltages. 

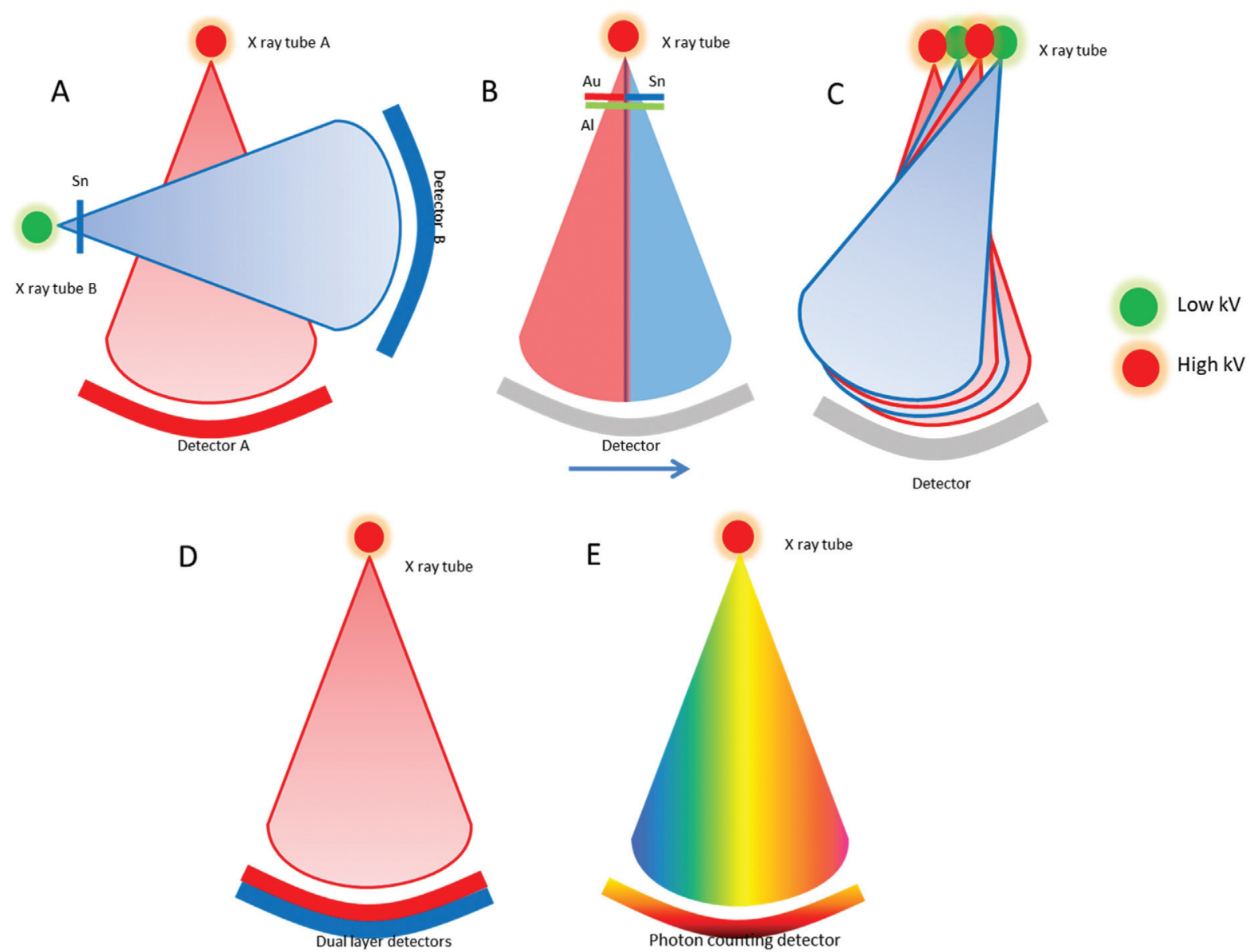

Fig. 1 Dual-energy computed tomography (DECT) techniques. (A) Dual-source DECT. Two X-ray tubes with corresponding detectors are mounted orthogonally at $90^{\circ}$ with different tube potentials. (B) Twin beam filtration. Single X-ray source with two different filters arranged along the Z-axis. (C) Fast kilovolt switching. X-ray tube rapidly switches between the low and high voltages in a short time ( $<0.2$ milliseconds) within a single gantry rotation. (D) Dual-layer detector. A single source and a layered or sandwich detector. (E) Photon counting devices.

\section{Limitation}

- Reduced spectral resolution due to the inability to modulate tube current and optimize spectral filtration.

\section{Twin Beam Filtration}

In this source-based approach, a split filter is placed in front of the X-ray tube in the collimator in a single source-detector configuration. It is composed of gold and tin stacked adjacent to each other in a longitudinal direction to achieve spectral separation into low- and high-energy beams, and the corresponding halves of the detector detect the low- and highenergy spectra. ${ }^{5,9}$ Tin and gold filter the polychromatic beam's low- and high-energy components, thereby increasing and decreasing the mean energy of the beam, respectively, leading to spectral separation. ${ }^{7,8}$ This is the technique launched by Siemens Healthineers, Germany.

\section{Advantages}

- Not much hardware modification is necessary, and hence, it is cost-effective. The addition of a split filter to the tube collimator of an existing CT scanner is done to perform dual-energy imaging. ${ }^{5,7}$ Image acquisition is possible in the whole FOV, and automatic tube current modulation optimizes radiation dose to patient. ${ }^{5,7}$

\section{Limitations}

- Spectral separation resulting from a split filter is limited, compared with that achieved in a dual-source dual-energy scanner. ${ }^{8,9}$

- There is also potential for cross-scattering, with one side of the beam contaminating the other side of the detector.

- In addition, a higher power of the X-ray tube is necessary due to the prefiltration of the beam, which is a constraint in imaging obese patients.

- There is temporal skew between low- and high-energy data. $^{5}$

\section{Sequential Acquisition}

This is one of the earliest approaches suggested for acquiring dual-energy images without the need for dedicated hardware. ${ }^{8}$ Imaging the entire volume is done sequentially in low and high kilovolts, but this results in a long delay between the two datasets. Alternating the tube potential between each successive gantry rotation can reduce this delay to some extent. ${ }^{2,5,9}$ 


\section{Advantages}

No significant hardware modification is needed-sequential scanning in any CT scanner in two different voltages is acquired, and the dataset can be combined for spectral analysis. $^{5}$

Imaging in two different potentials at the exact location during each gantry rotation provides the same view angle, leading to projection space alignment of the two datasets and, hence, projection-based material decomposition. ${ }^{7}$

Automatic exposure control can be implemented, enabling tube current modulation for different tube potentials providing optimal noise levels between the two potentials. $^{7}$

\section{Limitations}

- In sequential scanning, patient movement between the acquisitions can lead to spectral distortion.

- Sufficient temporal skew makes its implementation difficult in cardiac studies and angiographic acquisitions, requiring high temporal resolution. ${ }^{5}$ Thus, its use is limited to relatively static organs and non-contrast studies. $^{6,8}$

\section{Detector-Based Acquisition}

\section{Dual-Layer Detector}

This design consists of a single source providing a highenergy beam ( 120 or $140 \mathrm{kV}$ ) and a layered or sandwich detector. This detector consists of two layers with different scintillator materials, and as a result, there is maximal sensitivity for different photon energies. The inner or the top layer is made of zinc selenide or cesium iodide crystals which absorb the low-energy photons, and the outer or bottom layer is made of gadolinium oxysulfide, which is sensitive to high-energy photons. ${ }^{2,5,7,8}$ This technique, thus, exploits the polychromatic nature of the X-ray beam. ${ }^{5,8}$ The thickness of an individual layer is designed to enable comparable noise levels across the two energy datasets. ${ }^{2,9}$ This technology was established by Philips Healthcare, the Netherlands.

\section{Advantages}

Image acquisition can be performed at full FOV with no constraints in gantry rotation time, dose optimization techniques. ${ }^{7,8}$

Since spectral separation is achieved at the level of the detector, there is a perfect alignment of the low- and highenergy datasets with excellent temporal and spatial registration, and hence, material decomposition can be performed in the projection domain. ${ }^{5,7}$

The conventional dataset can be obtained by combining the low- and high-energy spectral data and reconstructed by standard techniques of iterative reconstruction and filtered back projection. $^{8}$

Scan acquisition is always performed in a dual-energy mode allowing for retrospective spectral analysis, especially useful in scenarios where there is no predetermined indication for dual-energy scan. ${ }^{5,7}$

\section{Limitations}

- Spectral analysis can be done only in scans performed with a higher energy beam ( 120 or $140 \mathrm{kV}) .^{7}$

- There is suboptimal spectral resolution and material decomposition due to overlap between the absorption properties of the scintillator material ${ }^{5,6}$ (leading to unsharp distinction between low- and high-energy photons). This can be overcome by introducing an interlayer filter between the two detector layers but at the cost of dose efficiency. ${ }^{5}$

- Photon interaction in one detector pixel can result in scattering and subsequent interaction in another pixel, known as cross-talk between the two detector layers. ${ }^{5,7}$

\section{Photon-Counting Detectors}

This design of spectral CT is the subject of ongoing research and has the potential for multi-energy imaging. A photoncounting detector (PCD) is an energy-resolving detector that can count discrete photon interactions. These detectors are highly specialized and are based on semiconductors such as cadmium telluride, cadmium zinc telluride They convert the incident photons into electrical signals whose magnitude depends on the energy of the incident X-ray photon. Pulse height discriminator analyses these electrical pulses and place them in energy bins based on specific energy thresholds. ${ }^{2,7,9}$ Electronic noise below the lowest energy threshold is wholly eliminated and, hence, does not affect the count rates. $^{7}$

\section{Advantages}

- Improved spectral resolution and increased dose efficiency.

- Higher geometrical efficiency than energy integrating detectors. $^{5}$

- K-edge imaging-used in material differentiation wherein higher atomic number elements can produce a k-edge effect, which enables their differentiation from other materials. This can potentially be used to differentiate iodine from gadolinium contrast agents and separation of iodine, gadolinium, and bismuth in multiphasic studies. ${ }^{5,7}$

- Inherent spatial and temporal resolution enables multiphasic acquisition and imaging of moving organs. ${ }^{7}$

\section{Limitations}

- Pulse pile-up effect occurs due to high X-ray flux in CT in the order of 109 per second per $\mathrm{mm}^{2}$. This happens when consecutive low-energy pulses at a short interval are incorrectly registered, leading to the loss of photon counts and increased image noise. .,9 $^{7}$

- Charge sharing occurs due to pulse sharing across the multiple detector pixels, leading to incorrect counts and decreased spectral resolution. 2,7

- K escape is the absorption of photon energies due to the k-edge effect and release of characteristic X-ray resulting in energy loss. ${ }^{2,7}$

- Count rate drift occurs due to crystal defects in the detector, causing the trapping of electrons and holes, 

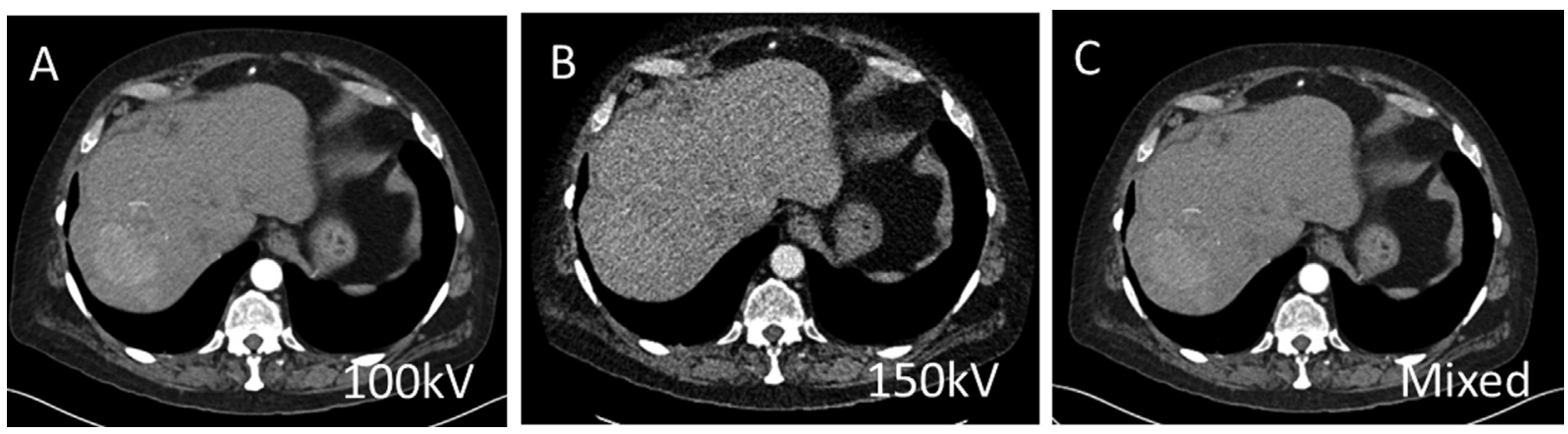

Fig. 2 Axial images of the contrast-enhanced CT of the abdomen at the same level in the arterial phase showing a hyper-vascular lesion in the right lobe of liver. (A) Low-energy (100 kV dataset), (B) high energy (150 kV dataset), and (C) mixed set.

leading to changes in signal pulse characteristics in detector elements, causing ring artifacts. ${ }^{7}$

- Cross-talk effect occurs when photon interaction occurs near the border of detector pixels. ${ }^{9}$

\section{DECT Images}

Mixed/Blended Set of Images

A single set of blended/mixed images is generated from the low- and high-energy datasets (-Fig. 2). The recommended blending ratio is 0.3 (70\% from the high-energy dataset and $30 \%$ from the low-energy dataset). The images generated are equivalent to the images of conventional $120 \mathrm{kVp}$ singleenergy data acquisition. The mixed images have the advantages of high contrast from the low-energy dataset and less noise from the high-energy dataset. These images can be generated easily without the requirement of complex postprocessing analysis. ${ }^{10}$

\section{Image-Processing Algorithms}

Post-processing in DECT is an essential technical aspect for acquiring data needed for a wide range of clinical applications. The processing of data, especially for material decomposition, can be performed either in the projection space (pre-reconstruction) or in the image space (postreconstruction).

The equivalent projection data obtained in the two energies are subtracted and reconstructed by filtered back-projection to obtain the spectral information in the projection space method. It is quantitatively more accurate, and beam hardening artifacts are eliminated in this method, though, in practice, they may not be removed entirely. In the image space approach, first, the reconstruction of the low- and high-energy datasets is done, and then, the difference in corresponding voxels in the two datasets is processed to obtain the spectral information. It is an easier to perform and more commonly used approach. It is preferred when there is a spatial or temporal inconsistency between the projection data, like in the case of dual-source scanners where there is a $90^{\circ}$ offset between the two datasets. ${ }^{2,6,7}$

\section{Material Decomposition}

DECT can decompose the material into its constituent elements based on energy and material dependence of X-ray attenuation. ${ }^{2}$ The basic mathematical algorithm to derive the material decomposition was first proposed in 1976 by Alvarez and Macovski, after which it had undergone subsequent alterations. ${ }^{8}$

The mass attenuation coefficient of a given material can be expressed as the linear combination of photoelectric and Compton effects, ignoring the $\mathrm{K}$ - or L-edge effects unless significant. It is calculated as the linear attenuation coefficient divided by the density of the absorber. Alternatively, any material attenuation coefficient can be given as a linear combination of attenuation coefficients of two basic materials present at different mass densities. For example, iodine and water are commonly used basic materials, and other materials can be expressed as the mixed or linear combination of iodine and water. Series of mathematical equations and derivations depicting the X-ray attenuation of the material in two energy spectra were devised, and as a result, mass density, atomic number, and material-specific information of given material were obtained. ${ }^{2,7-9}$

Material decomposition gives the amount of primary material in the image voxel required to produce the observed $\mathrm{X}$-ray attenuation rather than the actual composition of each image voxel. ${ }^{9}$

\section{Multimaterial Decomposition}

It decomposes a material in a given voxel into three or more basic materials depending on the need in specific clinical applications. This can be done if one of the materials has a K-edge effect, i.e., K-edge in the given energy range. The accuracy of material decomposition increases with more number of basic materials. ${ }^{8}$ However, noise levels in each energy spectra and the difference in the atomic number of basic materials can influence the accuracy of material decomposition. $^{2}$

\section{Material-Specific Images}

In material-specific images, the selected basic materials can be detected, estimated, or subtracted according to clinical needs and used in various clinical applications. The distribution of the given material's mass density can be mapped. Material-specific images of the commonly used basic materials, iodine and water, are called iodine and water maps, respectively. Materials like iodine and calcium can be removed from the voxels to generate virtual non-contrast (VNC) and virtual non-calcium 

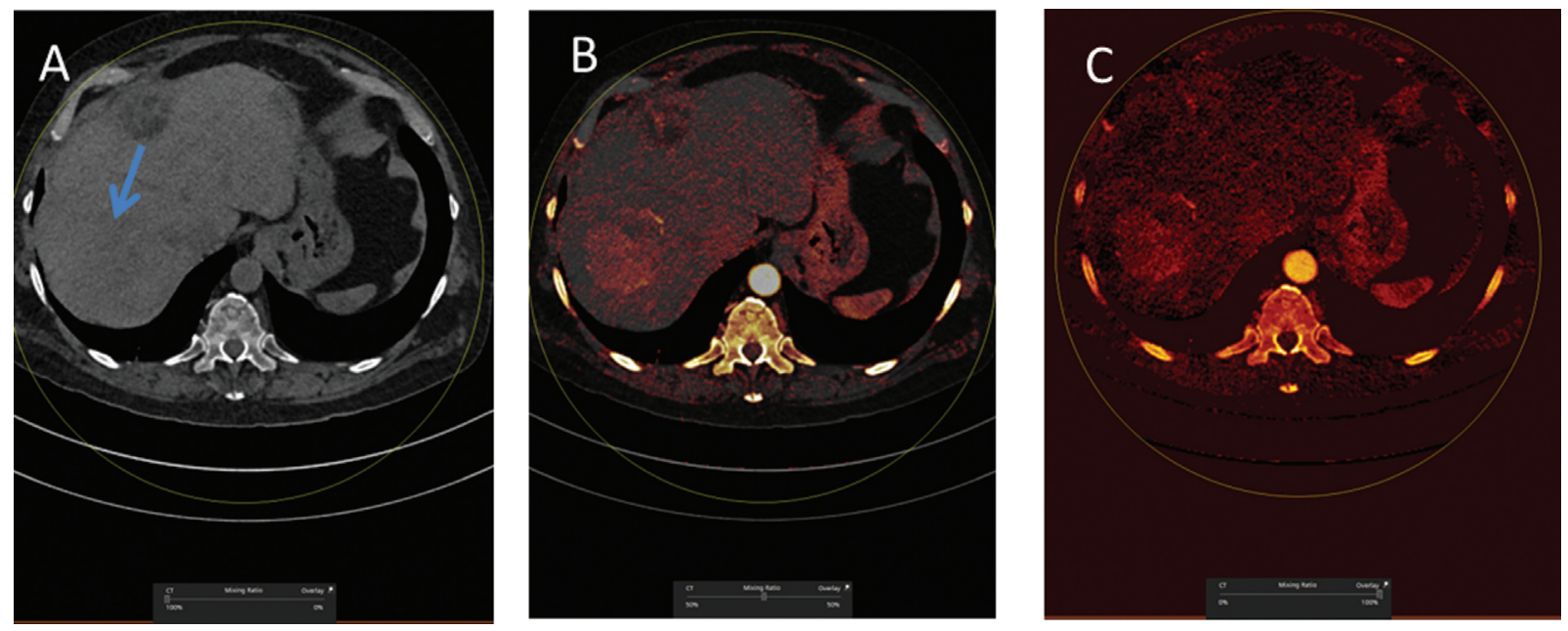

Fig. 3 Axial images of the contrast-enhanced CT of the abdomen at the same level in the arterial phase showing a hyper-vascular lesion in the right lobe of liver. (A) Virtual non-contrast image, with no iodine density in the region of lesion in the right lobe of liver (blue arrow). (B) lodine overlay image. (C) lodine map image.

images, respectively. ${ }^{7,8}$ However, the signal is also contributed by materials present other than the primary materials in a given voxel during material decomposition. For example, in iodine-water decomposition, there may be other materials like fat or bone, represented both in iodine-removed (VNC) and in water-removed images. Hence, there is a difference in the Hounsfield unit value of fat in the VNC image compared with a conventional non-contrast image. ${ }^{7}$ Increased noise, scatter, and beam hardening artifacts can cause error and decrease the accuracy of material separation. ${ }^{7}$

\section{Virtual Non-Contrast Images}

This is one of the potential applications of material-specific images in DECT, where the iodine component can be subtracted from the voxels in a contrast-enhanced scan to yield a VNC image ( - Fig. 3). VNC images are being evaluated as a substitute for a proper precontrast scan in a multiphasic acquisition and can exclude initial non-contrast scan resulting in radiation dose reduction. It can also be used as a problem-solving tool when only a contrast scan is acquired. A subtly increased attenuation is found: VNC can differentiate between subtle contrast enhancement and increased attenuation in soft tissue. ${ }^{2}$

\section{Virtual Monoenergetic Images}

Virtual monoenergetic images (VMI), also known as virtual monochromatic images, can be generated from dual-energy acquisition, and these portray the appearance of images when a monochromatic beam of X-rays is used. VMI has its roots in as early as the 1970s when Alvarez and Macovski suggested that CT images can be synthesized at any energy within diagnostic range and that the images have less beam hardening artifacts and more accurate attenuation measurements. 3,10

This is mainly based on the material decomposition of basic materials. As mentioned earlier, the linear attenuation coefficient can be given by the linear combination of the mass attenuation coefficient of two basic materials, and the mass attenuation coefficient can be expressed as a linear combination of photoelectric and Compton effects. A mass density map of each primary material at the two energies is obtained by solving the relevant equations. Monoenergetic images can then be synthesized from mass density and mass attenuation coefficients of basic materials at each energy. ${ }^{7,10}$ The usual energy range for VMI is 40 to $200 \mathrm{keV}$ (-Fig. 4), and the optimal contrast-to-noise ratio for iodine is obtained at the range of 40 to $70 \mathrm{keV}^{2,7}$

In low-kiloelectron-volt images, contrast enhancement is more pronounced so that even subtle enhancement can be assessed, and there is scope for reducing contrast dose. However, there is increased noise at lower energies compared with the higher energy range. High-kiloelectron-volt images reduce beam hardening artifacts, photon starvation, and metallic artifacts.

\section{Overview of Clinical Applications of DECT $^{2,7,8}$}

The DECT has many potential applications in the evaluation of abdominal pathologies and few of them are listed in - Table 1.

\section{Conclusion}

DECT has become the state-of-the-art technology in CT imaging because of its ability to discern the material composition and also to generate monoenergetic images using spectral information. It has now emerged as a powerful tool for faster and advanced imaging by opening a wide range of potential applications. Multi-energy imaging by PCD which is a subject of ongoing research holds promising and exciting possibilities. On the whole, DECT is a boon to modern imaging, marking a significant milestone in CT technology evolution. 

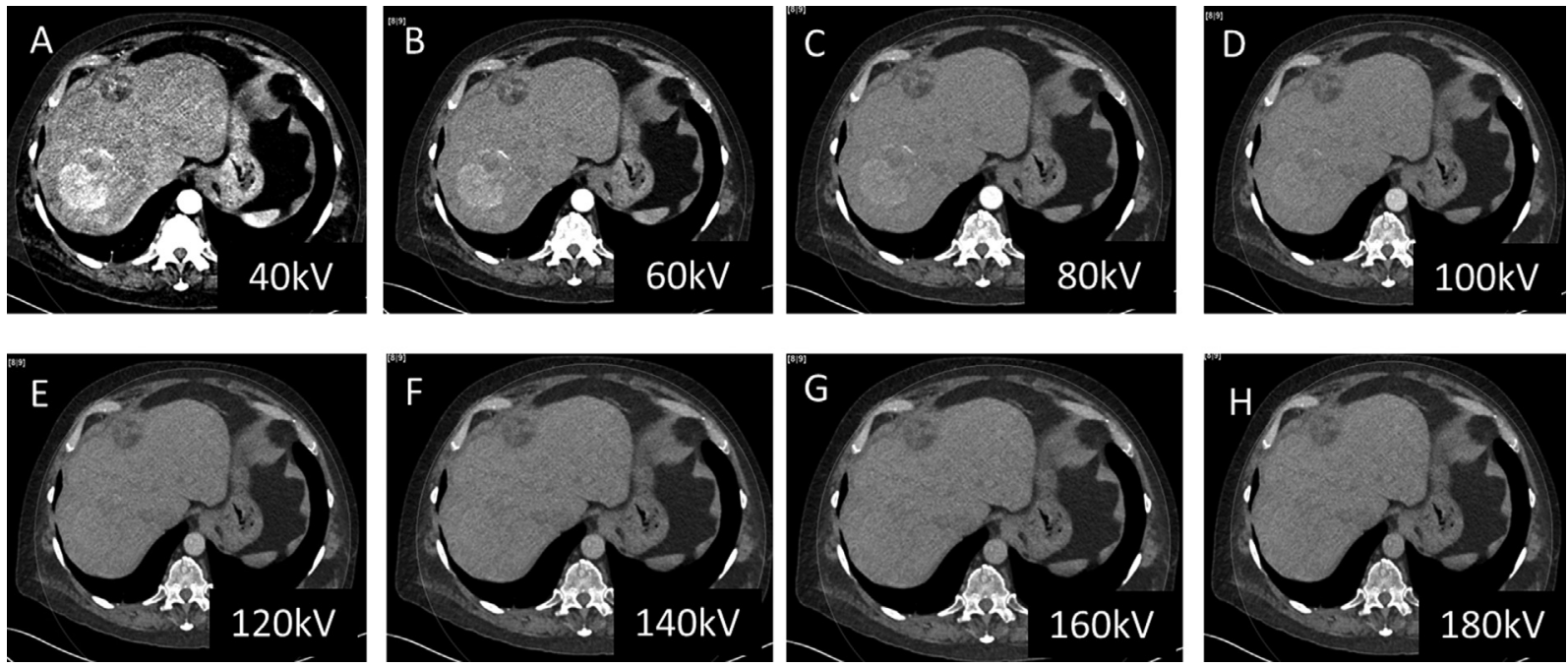

Fig. 4 (A-H) Virtual monoenergetic images. Axial images of the contrast-enhanced CT of the abdomen at the same level in the arterial phase showing a hyper-vascular lesion in the right lobe of liver in varying energy levels from 40 to $180 \mathrm{kV}$.

Table 1 Clinical applications of DECT

\begin{tabular}{|c|c|c|}
\hline & Algorithm & Clinical applications \\
\hline \multirow[t]{13}{*}{$\begin{array}{l}\text { Material } \\
\text { based }\end{array}$} & Material differentiation & $\begin{array}{l}\text { For differentiation between uric acid and non-uric acid stones based on the } \\
\text { atomic number. } \\
\text { Even subclassification among non-uric acid stones is possible. }\end{array}$ \\
\hline & & $\begin{array}{l}\text { Contrast enhancement and hemorrhagic lesions (iodine vs. iron) which may } \\
\text { have similar attenuation in single energy CT can be differentiated. }\end{array}$ \\
\hline & Material subtraction & $\begin{array}{l}\text { Virtual non-contrast images-identification and removal of iodine in voxels of } \\
\text { contrast-enhanced image so that the acquisition of separate non-contrast scan be } \\
\text { avoided and also to assess subtle increased attenuation in contrast images. }\end{array}$ \\
\hline & & $\begin{array}{l}\text { Virtual non-calcium images-identification and removal of calcium from bone } \\
\text { to assess bone marrow edema, providing an alternative for MRI. }\end{array}$ \\
\hline & & $\begin{array}{l}\text { Bone removal in CT angiography-identification and removal of bone enabling } \\
\text { the better visualization of contrast-enhanced vessels (- Fig. } \mathbf{5} \text { ). }\end{array}$ \\
\hline & & $\begin{array}{l}\text { Atherosclerotic plaque removal ( } \mathbf{- F i g .} \mathbf{5} \text { )-for the better visualization of } \\
\text { vessel lumen in maximum intensity projection }\end{array}$ \\
\hline & Material quantification-iodine & $\begin{array}{l}\text { can be used to assess tumor for treatment response after } \\
\text { locoregional/systemic therapy }\end{array}$ \\
\hline & & Improve detection and delineation of solid and cystic lesions in pancreas. \\
\hline & & Differentiation of bland and tumor thrombus \\
\hline & & For the identification of bowel ischemia \\
\hline & & useful in detection in the area of active bleeding in GI bleed \\
\hline & & Helpful in differentiating hyperdense enteric content from iodine uptake. \\
\hline & Material quantification-fat/iron & Quantitative evaluation of fatty liver (-Fig. 6) /hemochromatosis \\
\hline \multirow[t]{5}{*}{$\begin{array}{l}\text { Energy } \\
\text { based }\end{array}$} & $\begin{array}{l}\text { Virtual monochromatic images } \\
\text { (VMI)-low-kiloelectron-volt images }\end{array}$ & $\begin{array}{l}\text { Have pronounced contrast enhancement and, hence, used for improving the } \\
\text { detection of hyper-vascular liver lesions/small neuroendocrine tumors in the } \\
\text { pancreas. }\end{array}$ \\
\hline & & Hypo-vascular liver lesions in the portal venous phase \\
\hline & & Improves the detection of non-radiopaque gall stones. \\
\hline & VMI-high-kiloelectron-volt images & $\begin{array}{l}\text { can be used for the reduction of beam hardening and metallic artifacts in } \\
\text { cases with orthopaedic prosthesis, dental implants, aortic stents aneurysmal } \\
\text { clips, etc. }\end{array}$ \\
\hline & Rho-Z method & Non-calcific gall stone \\
\hline
\end{tabular}

Abbreviations: DECT, dual-energy computed tomography; Gl, gastrointestinal. 

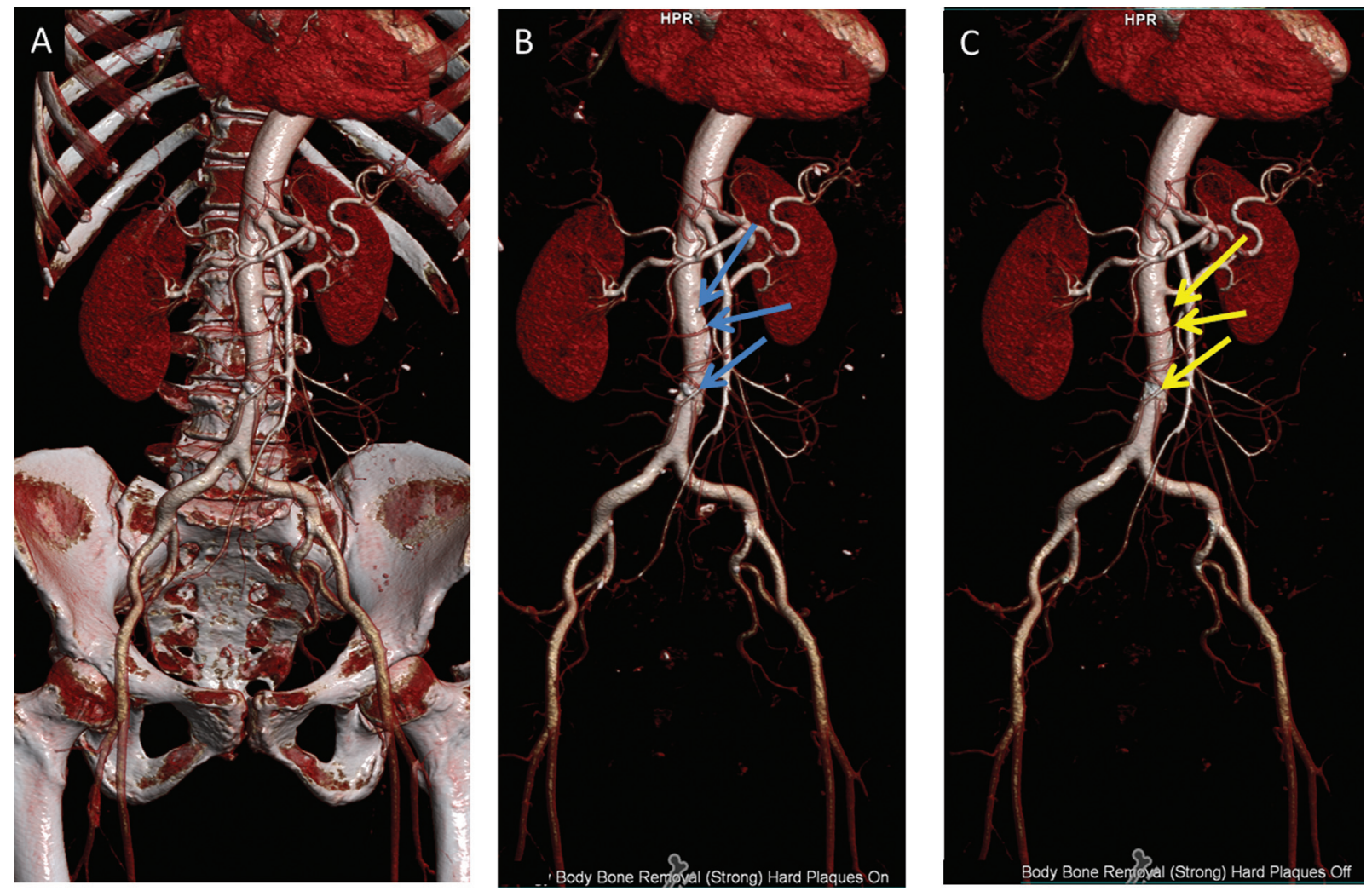

Fig. 5 Volume-rendered images of abdominal Aortogram. (A) Without the removal of bones, (B) with the removal of bones but with hard plaques on (blue arrow), and (C) the removal of bone and hard plaques (yellow arrow demonstrates the absence of plaques).
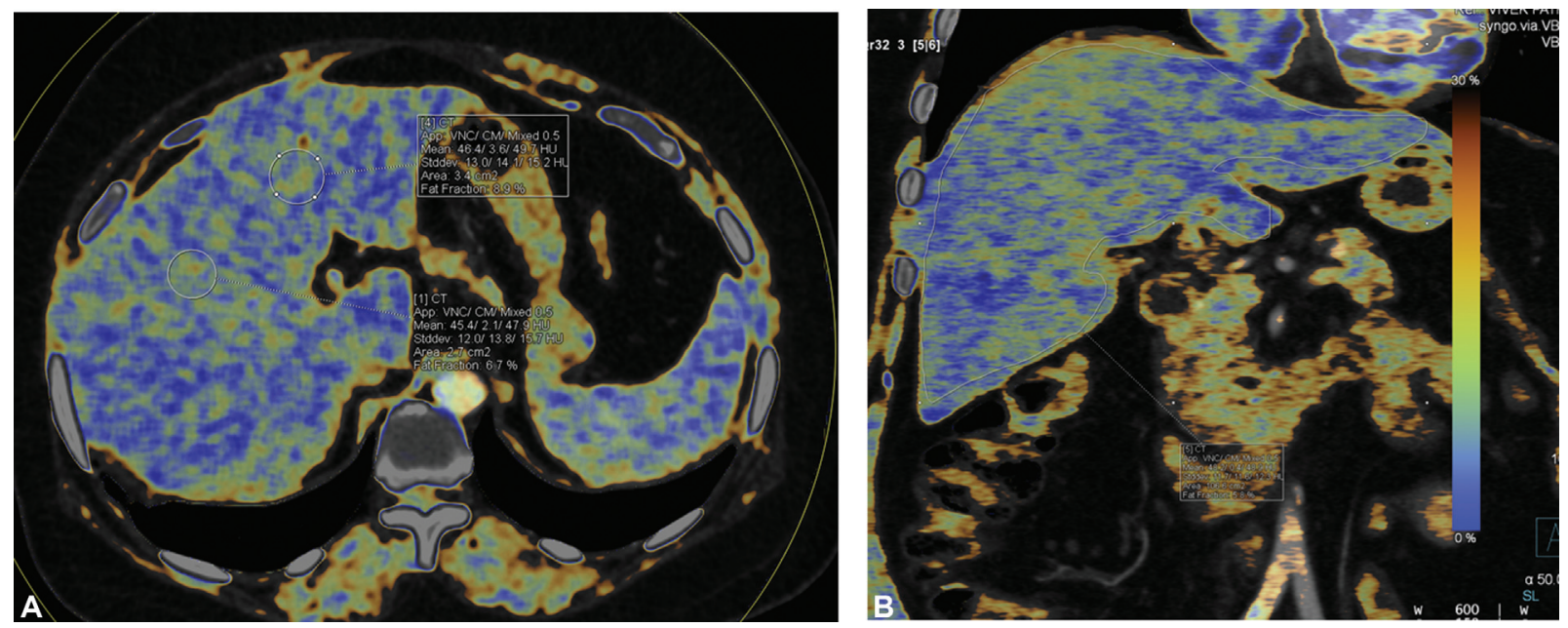

Fig. 6 Fat quantification images generated from the dual-energy computed tomography in (A) axial and (B) coronal planes. Circle region of interest (ROI) in axial and free-hand ROI in coronal images show fat fraction.

Conflict of Interest

None declared.

\section{References}

1 Kohl G. The evolution and state-of-the-art principles of multislice computed tomography. Proc Am Thorac Soc 2005;2(06):470-476, 499-500

2 McCollough CH, Leng S, Yu L, Fletcher JG. Dual- and multi-energy CT: principles, technical approaches, and clinical applications. Radiology 2015;276(03):637-653
3 Alvarez RE, Macovski A. Energy-selective reconstructions in X-ray computerized tomography. Phys Med Biol 1976;21(05):733-744

4 Macovski A, Alvarez RE, Chan JL-H, Stonestrom JP, Zatz LM. Energy dependent reconstruction in X-ray computerized tomography. Comput Biol Med 1976;6(04):325-336

5 Forghani R, De Man B, Gupta R. Dual-energy computed tomography: physical principles, approaches to scanning, usage, and implementation: part 1. Neuroimaging Clin N Am 2017;27(03): 371-384

6 Johnson TR. Dual-energy CT: general principles. AJR Am J Roentgenol 2012;199(5 Suppl)S3-S8 
84 Principles and Available Hardware in DECT Alavandar et al.

7 McCollough $\mathrm{CH}$, Boedeker K, Cody D, et al. Principles and applications of multienergy CT: report of AAPM task group 291. Med Phys 2020;47(07):e881-e912

8 Agostini A, Borgheresi A, Mari A, et al. Dual-energy CT: theoretical principles and clinical applications. Radiol Med (Torino) 2019;124 (12):1281-1295
9 So A, Nicolaou S. Spectral computed tomography: fundamental principles and recent developments. Korean J Radiol 2021;22 (01):86-96

10 Yu L, Leng S, McCollough CH. Dual-energy CT-based monochromatic imaging. AJR Am J Roentgenol 2012;199(5, Suppl): S9-S15 